\title{
An algebraic stability test for fractional order time delay systems
}

\author{
Münevver Mine Özyetkin ${ }^{a^{*}}$ and Dumitru Baleanu ${ }^{b, c}$ \\ ${ }^{a}$ Department of Electrical and Electronics Engineering, Aydin Adnan Menderes University, Turkey \\ ${ }^{b}$ Department of Mathematics, Faculty of Art and Sciences, Cankaya University, Turkey \\ ${ }^{c}$ Institute of Space Sciences, P.O. Box 07r125, Magurele-Bucharest, Romania \\ m.ozyetkin@adu.edu.tr,dumitru@cankaya.edu.tr
}

\section{ARTICLE INFO}

Article History:

Received 29 March 2019

Accepted 16 December 2019

Available 28 January 2020

Keywords:

Fractional order

Integer order

Time delay

Stability

AMS Classification 2010:

34H05; $93 \mathrm{C} 99$

\author{
ABSTRACT
}

In this study, an algebraic stability test procedure is presented for fractional order time delay systems. This method is based on the principle of eliminating time delay. The stability test of fractional order systems cannot be examined directly using classical methods such as Routh-Hurwitz, because such systems do not have analytical solutions. When a system contains the square roots of $s$, it is seen that there is a double value function of $s$. In this study, a stability test procedure is applied to systems including $\sqrt{s}$ and/or different fractional degrees such as $s^{\alpha}$ where $0<\alpha<1$, and $\alpha \in \mathbb{R}$. For this purpose, the integer order equivalents of fractional order terms are first used and then the stability test is applied to the system by eliminating time delay. Thanks to the proposed method, it is not necessary to use approximations instead of time delay term such as Padé. Thus, the stability test procedure does not require the solution of higher order equations.

\section{Introduction}

The systems shown by differential equations with real orders instead of integer orders are called fractional order systems [1. Fractional order systems (FOS) are one of the most popular research topics of today. Although the mathematical analysis of such systems has been known since 1695, mostly, it has been discussed and investigated by mathematicians because of its complexity [1]. The most important feature of this subject is that it expresses real systems better than integer order ones [2]. As it is known time delays are intrinsic of a variety of electrical, electronic, and communication systems, control applications, power systems with long transmission lines, and many real world applications [3 5]. In control applications, there are many examples of neutral-type time-delay systems as well as discrete-continuous hybrid systems regarded as delay differential algebraic equations (DDAEs) 4]. Besides, power systems with long transmission lines can be modeled as DDAEs for certain assumptions 4]. Systems with neutral delay differential equations (NDDEs) contains delays in both the state variables and their time derivatives [3. If fractional order systems include time delay, the analysis of such systems becomes more and more complicated. And, the studies to obtain analytical solutions of fractional order systems with delays are very restricted.

Many studies have been carried out in relation to FOS, in the literature [6-14]. Analytical stability test procedures of FOS are still important research topics. Analytical stability test procedures such as the Routh-Hurwitz method cannot be applied to FOS,directly. There are some studies on the stability of FOS in [1,15] 22]. In [15], a method for stability analysis of distributed parameter systems having delay is presented. This method is also applicable to FOS. In [18, internal and external stabilities of fractional differential systems in the state-space form are investigated. In 16, stability for a certain class of linear and nonlinear fractional order systems is presented. A

*Corresponding Author 
test procedure based on the Nyquist stability criterion is presented in [1. However, the studies related to the stability of FOS continue, and an analytical stability test technique does not exist, to the best of the author's knowledge. In addition, examining of time response analysis of such systems is very complicated since calculating inverse Laplace transforms of them is difficult, in spite of this, time response analysis of FOS can be made by using integer order approximations. Some toolboxes designed to examine such systems can be found in $[23-26]$. The frequency domain based methods can be considered advantageous for FOS since the stability of FOS can be tested thanks to frequency domain methods such as the Nyquist curve.

In the literature, the most preferred method to investigate FOS is to use integer-order approximations [27,28]. That is, in order to apply methods in classical control to such systems, integer-order equivalent transfer functions can be used. There are many approximation methods to obtain integer order equivalencies of fractional order differential equations. For example, the continued fraction expansion method (CFE), Oustaloup, Carlson and Matsuda's method and Maclaurin series etc. [29]. In this study, the CFE method is preferred to obtain integer order approximations of FOS. Then, the proposed algebraic stability test is applied to the system. According to results that are obtained in [28], it is observed that when the degree of used approach increases the obtained results are closer to the original system. However, this makes the process mathematically more complicated. As aforementioned, the analytical stability test of FOS cannot be performed by classical methods, directly. Therefore, this study is aimed to fill this gap. For this purpose,in the first step, the integer order equivalents of fractional order terms are used. In the second step, the stability test is applied to the system by eliminating time delay. As it is known, in general, analytical stability test procedures of time delay systems require to use some approximation methods such as Padé. Besides, we need to use higher order Padé approximations instead of time delay term to obtain more reliable results. This process makes the analysis of time delay systems more complicated. However, using the proposed method, it is not necessary to use approximations instead of time delay term since it is eliminated. Thus, the stability test procedure does not require the solution of higher order equations. This makes the proposed method practical and preferable. For the future studies, this method can be extended for systems with multiple time delays. It can also be applied to systems controlled by fractional order controllers. Besides, power systems modeled by delayed differential equations can be investigated by using the proposed method.

This paper is organized as follows: In the first section, literature information has been presented. In the second section, fractional order time delay systems are introduced. In the third section, an algebraic stability test procedure is presented for fractional order time delay systems. In the last section, concluding remarks have been presented.

\section{Fractional order time delay systems}

Systems where derivatives are expressed in fractional orders instead of integer ones are called fractional order systems. A unity feedback control system is given in Fig.(11).

Definition 1. Fractional order time delay systems are represented as follows.

$$
\begin{aligned}
& G(s)=\frac{N(s)}{D(s)} e^{-h s} \\
& =\frac{b_{m} s^{\beta}+b_{m-1} s^{\beta} m-1+\ldots+b_{0} s^{\beta_{0}}}{a_{n} s^{\alpha_{n}}+a_{n-1} s^{\alpha_{n-1}+\ldots+a_{0} s^{\alpha_{0}}}} e^{-h s}
\end{aligned}
$$

Where, $h$ represents time delay, $a_{k}(k=0, \ldots, n)$, and $b_{k}(k=0, \ldots, m)$ are constants, $\alpha_{k}(k=$ $0, \ldots, n)$, and $\beta_{k}(k=0, \ldots, m)$ are arbitrarily real numbers. Where, one can assume inequalities $\alpha_{n}>\alpha_{n-1}>\ldots>\alpha_{0}$ and $\beta_{m}>\beta_{m-1}>\ldots>\beta_{0}$ without loss of generality [30].

Time delay, which may cause poor performance or even instability in system response, is a common case in many industrial processes. It can be originated from the internal dynamics of the system [31]. Since stability test of time delay systems cannot be performed directly, some approximations such as Padé are used instead of time delay term. However, in some cases, the first order Padé approximation may not give correct results in terms of stability [31]. Therefore, to obtain more correct results, it is necessary to increase the degree of approach, which makes the processes mathematically more complicated. Thus, a stability test eliminating time delay will be important for simplicity.

\section{A Stability test for fractional order time delay systems}

Definition 2. The characteristic equation for a linear system having a single time delay is expressed as follows, where $h$ is time delay [32].

$$
\Delta(s, h)=\Delta_{1}(s)+\Delta_{2}(s) e^{-h s}=0
$$




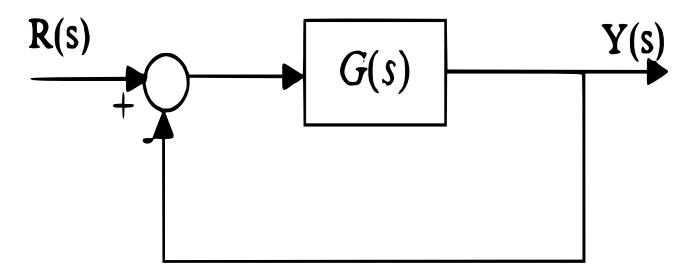

Figure 1. A feedback system.

Definition 3. The general representation of this expression for systems having multiple commensurate time delay is given as follows [32].

$$
\Delta_{n}(s, h)=\sum_{k=0}^{n} \Delta_{k}(s) e^{-k s h}
$$

For zero delay systems, the necessary and sufficient condition of asymptotic stability is known as the presence of all the roots of the characteristic equation on the left half of the complex s plane. For systems with time delay, this result may be stable or unstable for some values of $h$ [32. It has been concluded that the system is asymptotically stable regardless of delay for a particular case where all positive values of time delay $h$ are not negative [32]. Here, the main problem is to determine $h$ values when $\Delta(s, h)=$ $\Delta_{1}(s)+\Delta_{2}(s) e^{-h s}=0$ has root/or roots on the complex axis. $\Delta(s, h)=0$ is an implicit function of $s$ and $h$, which may exceed or not exceed the imaginary axis. Suppose that all the roots of $\Delta(s, 0)=0$ are in the left-half plane. So, the system is stable for zero time delay. If $\Delta(s, h)=0$ has a root on the imaginary axis when $s=j \omega$ for some values of $h$, it can be said that $\Delta(-s, h)=0$ has also a root on the imaginary axis for the same values of $h$ and $\omega$. Thus, with the same common root $\Delta(s, h)=0$ and $\Delta(-s, h)=0$ for the determination of the roots on the imaginary axis, we do not need to find $h$ values. That is, a structure independent of time delay is obtained.

Theorem. A system is asymptotically stable regardless of delay for a particular case where all positive values of time delay $h$ are not negative. If $\Delta(s, h)=\Delta_{1}(s)+\Delta_{2}(s) e^{-h s}=0$ has root $/$ roots on the complex axis, the value range of $h$ is calculated for the stability.

Proof. $\Delta(s, h)=0$ is an implicit function of $s$ and $h$, which may exceed or not exceed the imaginary axis. If all the roots of $\Delta(s, 0)=0$ are in the left-half of $s$ plane, the system is stable for zero time delay. If $\Delta(s, h)=0$ has a root on the imaginary axis when $s=j \omega$ for some values of $h, \Delta(-s, h)=0$ has also a root on the imaginary axis for the same values of $h$ and $\omega$. Thus, with the same common root $\Delta(s, h)=0$ and $\Delta(-s, h)=0$ for the determination of the roots on the imaginary axis, it is not necessary to find $h$ values.

Corollary 1. The Eq.(6) is obtained by eliminating the time delay $h$ from Eq.(44) and Eq.(5). It is clear that this structure is independent of time delay.

$$
\begin{gathered}
\Delta(j \omega, h)=\Delta_{1}(j \omega)+\Delta_{2}(j \omega) e^{-j \omega h}=0 \\
\Delta(-j \omega, h)=\Delta_{1}(-j \omega)+\Delta_{2}(-j \omega) e^{+j \omega h}=0 \\
M\left(\omega^{2}\right)=\Delta_{1}(j \omega) \Delta_{1}(-j \omega)-\Delta_{2}(j \omega) \Delta_{2}(-j \omega)=0
\end{gathered}
$$

Corollary 2. Where, it is clear that $M\left(\omega^{2}\right)$ is a polynomial in the form $\omega^{2}=-s^{2}$. If $M\left(\omega^{2}\right)=0$ does not have positive roots, the system is stable for all $h \geq 0$.

The proposed stability test procedure is summarized as follows:

(1) In the first step, Eq.(2) is turned into the form of Eq.(7) for $h=0$.

$$
\Delta(s, 0)=\Delta_{1}(s)+\Delta_{2}(s)=0
$$

It is tested whether the zeros of the characteristic equation are in the left half of $s$ plane. The system is stable for $h$ if all the zeros are located in the left half of $s$ plane. If so, the second step is applied.

(2) The presence of positive roots of $M\left(\omega^{2}\right)=$ 0 is investigated. The system is stable for all $h \geq 0$ if $M\left(\omega^{2}\right)=0$ does not have any positive roots. If $M$ has at least one positive root, the range of $h$ must be investigated for the stability.

(3) If the second condition is met, the following equations are used to determine the range of $h$ [32].

$$
\begin{aligned}
& \cos (\omega h)=\operatorname{Re}\left\{\frac{-\Delta_{1}(j \omega)}{\Delta_{2}(j \omega)}\right\}, \\
& \sin (\omega h)=\operatorname{Im}\left\{\frac{\Delta_{1}(j \omega)}{\Delta_{2}(j \omega)}\right\}
\end{aligned}
$$


For the given value of $\omega, h_{0}(\omega)$ is the smallest positive value of $h$ providing Eq. (8), and the general solution is given as follows.

$$
h=h_{0}(\omega)+2 \pi n / \omega, n=0,1,2,3, \ldots
$$

More information about the method can be found in 32,33 . Let handle some examples to better understand the subject.

\subsection{Example 1}

Consider the characteristic equation given as follows.

$$
\Delta(s, h)=s^{2}+4 s+4-e^{-h s}=0
$$

When the procedure described above is applied, one obtains

$$
\Delta(s, 0)=s^{2}+4 s+3=0
$$

for $\Delta(s, 0)=0$, we obtain $s=-3$ and $s=-1$. It is clear that the system is stable for $h=0$. Thus, the second step is applied to the system as follows.

$$
\begin{aligned}
& M\left(\omega^{2}\right)=\left(-\omega^{2}+4 j \omega+4\right)\left(-\omega^{2}-4 j \omega+4\right) \\
& -1=\omega^{4}+8 \omega^{2}+15
\end{aligned}
$$

Since $\omega^{2}=-3$ and $\omega^{2}=-5$, there is no positive root of $M\left(\omega^{2}\right)=0$. It means that there is no any point touching or crossing imaginary axis. Thus, this system is stable independent of time delay $h$.

\subsection{Example 2}

For a unity feedback system given in Fig.(11), $G(s)$ is given by Eq. (13).

$$
G(s)=\frac{1}{s^{1.1}+2} e^{-h s}
$$

The characteristic equation of the system is obtained as follows.

$$
\Delta(s, h)=s^{1.1}+2+e^{-h s}=0
$$

In this equation, if we use the first order integer approximation instead of fractional order term, the characteristic equation is obtained as follows.

$$
\Delta(s, h)=s^{2}+2.46 s+2+(0.82 s+1) e^{-h s}=0
$$

The characteristic equation is stable for $h=0$ and it is obtained as follows.

$$
\Delta(s, 0)=s^{2}+3.28 s+3=0
$$

In this case, the second stage is applied. Therefore, $M$ is obtained by Eq.(17)

$$
\begin{aligned}
& M\left(\omega^{2}\right)=\left(-\omega^{2}+2.46 j \omega+2\right)\left(-\omega^{2}-2.46 j \omega+2\right) \\
& -(1+0.82 j \omega)(1-0.82 j \omega)
\end{aligned}
$$

Since Eq.(17) does not have a positive solution, the system is stable regardless of time delay. Let's examine the Nyquist curve of the system to confirm this result. The Nyquist diagrams of the original system and the first order approximation for $\omega=0: 0.01: 5$, and $h=1$ are shown in Fig.(21). As can be seen from the Fig.(2), the system is stable because the curve does not contain the critical point $(-1, j 0)$. Besides, the results of original system and of first order approximation are very close to each other. In Fig.(3), the Nyquist diagrams of the original system are given for $\omega \in[0,50]$, and $h=0.1: 0.1: 2$. The Nyquist diagrams of original system and of first order approximation for $\omega \in[0,50]$, and $h=0.1: 0.1: 2$ are shown in Fig.(4). As can be seen from Fig.(3) and Fig.(4), the curves do not include the critical point for increasing values of $h$. Therefore, if the system is stable for condition 1 and 2, as stated in the stability test procedure in section 3 , it can be said that it is stable for all values of $h \geq 0$. The Nyquist diagrams are shown in Fig.(3) and Fig.(44) also support this result.

In this example, if we use second order integer approximation instead of fractional order term, the characteristic equation is obtained as follows.

$$
\begin{aligned}
& \Delta(s, h)=1.351 s^{3}+6.67 s^{2}+10.34 s+2.702 \\
& +\left(s^{2}+4.67 s+1.351\right) e^{-h s}=0
\end{aligned}
$$

If the procedure is applied to the system, one obtains

$$
\Delta(s, 0)=1.351 s^{3}+7.67 s^{2}+15.01 s+4.053=0
$$

where, $s_{1,2}=-2.6791 \pm j 1.491$, and $s_{3}=-0.3191$. It is clear that the system is stable for $h=0$.

In the second step, $M$ is obtained by Eq.(20)

$$
\begin{aligned}
& M\left(\omega^{2}\right)=\left(-1.351 j \omega^{3}-6.67 \omega^{2}+10.34 j \omega+2.702\right) \\
& \times\left(1.351 j \omega^{3}-6.67 \omega^{2}-10.34 j \omega+2.702\right) \\
& -\left(-\omega^{2}+4.67 j \omega+1.351\right)\left(-\omega^{2}-4.67 j \omega+1.351\right)
\end{aligned}
$$

Since Eq.(20) does not have a positive solution, the system is stable regardless of time delay. Fig.(5) shows unit step responses of the system (using first order approximation) with the second order Padé approximation for $h=0.1: 0.1: 1.2$.

If we use a PI controller of the form $C(s)=$ $\left(k_{p} s+k_{i}\right) / s$, as shown in Fig.(6), unit step responses of the system are depicted in Fig.(17) for $\mathrm{k}_{p}=\mathrm{k}_{i}=1$. Here, It should be noted that $M$ can 


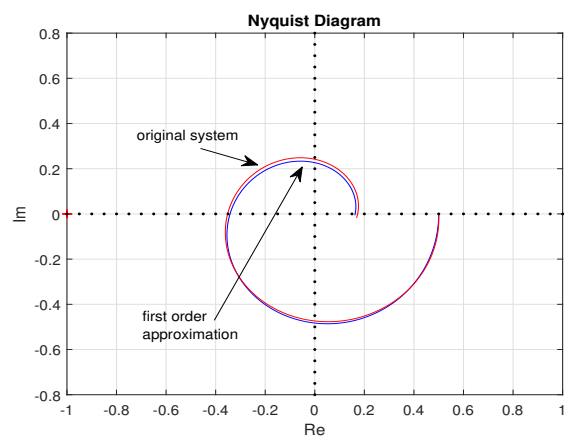

Figure 2. Nyquist diagrams of the original system and the first order approximation for $\omega \in[0,5]$ and $h=1$.

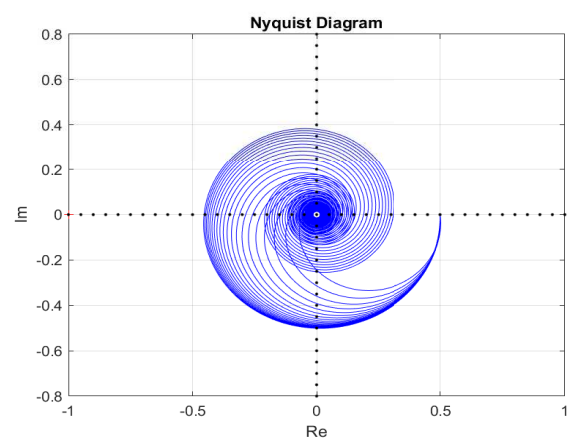

Figure 3. Nyquist diagrams of the original system for $\omega \in[0,50]$ and $h=0.1: 0.1: 2$.

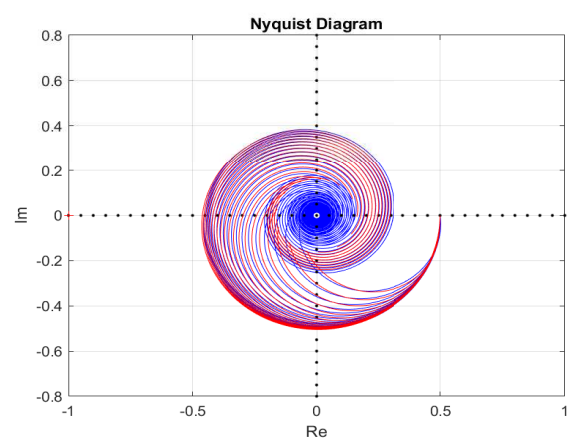

Figure 4. Nyquist diagram of the original system (blue) and the first order approximation (red) for $\omega \in[0,50]$ and $h=0.1: 0.1: 2$.

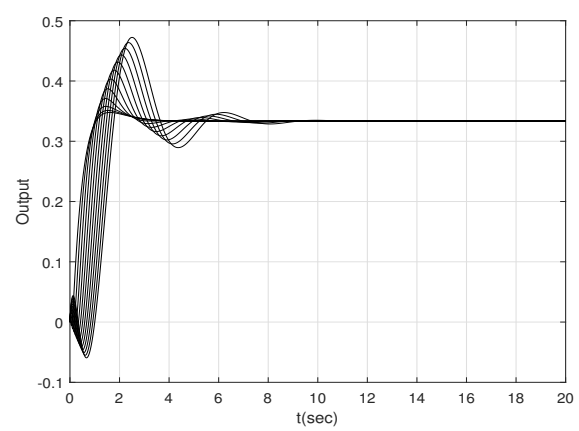

Figure 5. Unit step responses of the system (the first order approximation) with the second order Pade for $h=0.1: 0.1: 1.2$.

have positive solutions. Thus, delay free system can be unstable for some values of $\mathrm{k}_{p}$ and $\mathrm{k}_{i}$. In this case, it should be determined if the root touches the imaginary axis. If not, it means the system is unstable for $h=0$, but it is stable for infinite small $h$, that is, it is stable for 


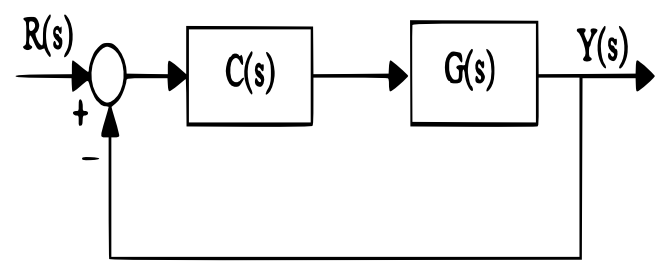

Figure 6. Feedback control system with PI controller.

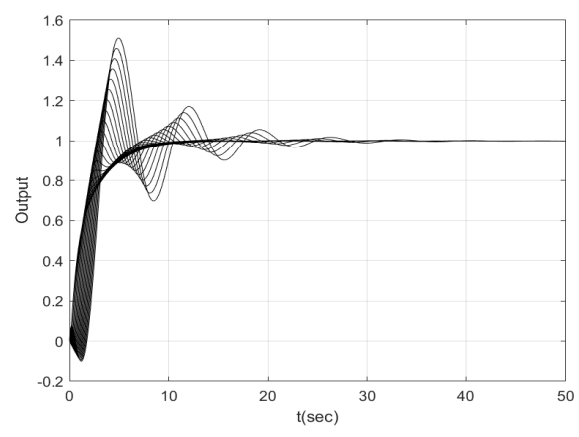

Figure 7. Unit step responses of the first order approximation and the second order Padé for $h=0.1: 0.1: 1.2$, and $\mathrm{k}_{p}=\mathrm{k}_{i}=1$.

$0<h<h_{0}(\omega)$. If the root touches the imaginary axis, then the system is unstable for $h=0$ and corresponding values of $k_{p}$ and $k_{i}$ for that $h$.

\subsection{Example 3}

In this example, $G(s)$ is given by

$$
G(s)=\frac{1}{\sqrt{s}(s+1)} e^{-h s}
$$

By using the first order approximation, the characteristic equation of the system is obtained as

$$
\Delta(s, h)=3 s^{2}+4 s+1+(s+3) e^{-h s}=0
$$

The characteristic equation for $h=0$ is obtained as follows

$$
\Delta(s, 0)=3 s^{2}+5 s+4=0
$$

Where the roots of the characteristic equation are $s_{1,2}=-0.833 \pm j 0.8$. Thus, the system is stable for $h=0$.

Thus, the second step of the procedure is applied to the system. And one obtains

$$
\begin{aligned}
& M\left(\omega^{2}\right)=\left(-3 \omega^{2}+4 j \omega+1\right)\left(-\omega^{2}-4 j \omega+1\right) \\
& -(3+j \omega)(3-j \omega)
\end{aligned}
$$

From Equation Eq.(24), we obtain $\omega^{2}=-1.5672$, and $\omega^{2}=0.5672$. Since $M\left(\omega^{2}\right)=0$ has a positive solution, there is a root touching the imaginary axis. In this case, it is necessary to determine the stability range of $h$. For this purpose, using the
Eq.(8) and Eq.(9) the range of $h$ making the system stable is calculated as $0 \leq h<2.1086$. The Nyquist curve for $h=1$ and the critical point $h=2.1086$ are shown in Fig.(8) according to the first order approximation. As can be seen from the Fig.(8), $h=2.1086$ is the critical point for stability of the system. However, this value was obtained according to the first order approximation. That is, when the second, third and fourth order approximations are used, the range value of $h$ would change. Depending on results in [28], it can be said that third and fourth order approximations provide the best results in capturing the original system. Thus, the value of $h$ obtained using these approximations will probably provide the best results for the system. But this change may not involve big numerical differences. That is, using first order approximation may be sufficient to examine stability of the system in terms of simplicity. The unit step responses of the system according to the first order approximation are given in Fig.(9) for $h=1$, and according to the critical point $h=2.1086$. As can be seen from the Fig.(9), the critical point gives an oscillatory response as expected. Fig.(10) shows the unit step responses of the system for $h=2.2$ and $h=2.5$ values exceeding the critical point. The system becomes unstable after the critical point.

In this example, if we use the second order integer approximation instead of the fractional order parameter, the characteristic equation is obtained as follows. 


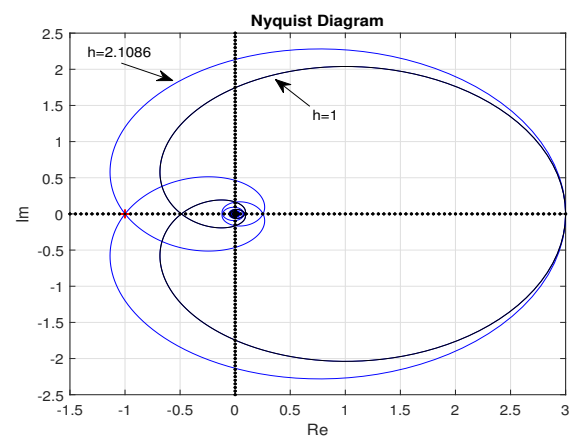

Figure 8. Nyquist diagram of the first order approximation for $h=1$ and the critical point $h=2.1086$.

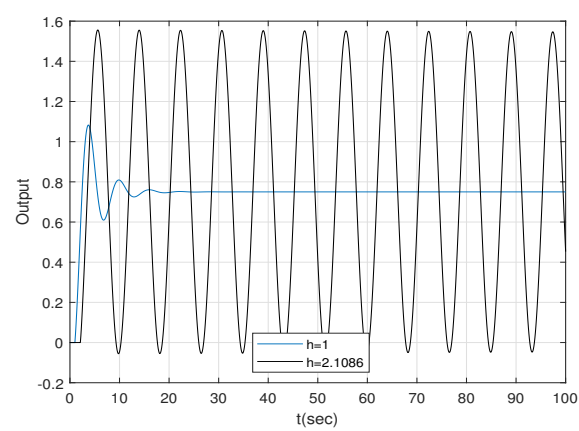

Figure 9. Unit step responses of Example 3.

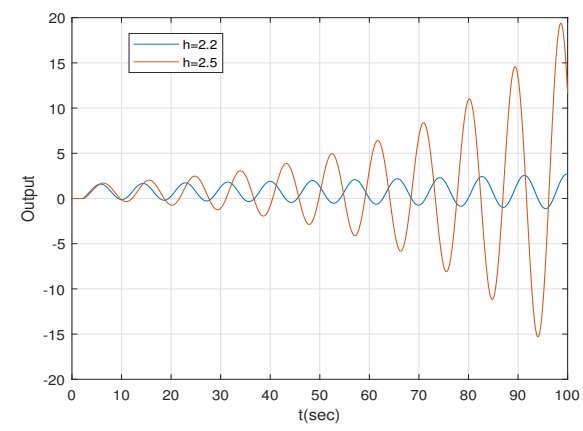

Figure 10. Unit step responses of Example 3 for $h=2.2$ and $h=2.5$.

$$
\begin{aligned}
& \Delta(s, h)=5 s^{3}+15 s^{2}+11 s+1 \\
& +\left(s^{2}+10 s+5\right) e^{-h s}=0
\end{aligned}
$$

If the procedure is applied to the system, one obtains

a.

$$
\Delta(s, 0)=5 s^{3}+16 s^{2}+21 s+6=0
$$

where, $s_{1,2}=-1.4074 \pm j 1.0654$, and $s_{3}=$ -0.3851 . It is seen that the system is stable for $h=0$.

b. In the second step, $M$ is obtained by Eq.(27)

$$
\begin{aligned}
& M\left(\omega^{2}\right)=\left(-5 j \omega^{3}-15 \omega^{2}+11 j \omega+1\right) \\
& \times\left(5 j \omega^{3}-15 \omega^{2}-11 j \omega+1\right)-\left(-\omega^{2}+10 j \omega+5\right) \\
& \times\left(-\omega^{2}-10 j \omega+5\right)
\end{aligned}
$$

From Equation Eq.(27), we obtain $\omega^{2}=-4.5038$, $\omega^{2}=-0.4906$, and $\omega^{2}=0.4344$. That is, $M\left(\omega^{2}\right)=0$ has a positive solution. Using the Eq.(8) and Eq.(9) the range of $h$ making the system stable is calculated as $0 \leq h<2.6962$. The unit step response of the system according to the second order approximation is given in Fig. (11) for the critical point $h=2.6962$. As can be seen from the Fig. (11), the critical point gives an oscillatory response as expected.

\section{Conclusion}

In this study, an algebraic stability test procedure based on the principle of eliminating time delay is presented for fractional order systems with a single time delay. Thus, mathematical operations 


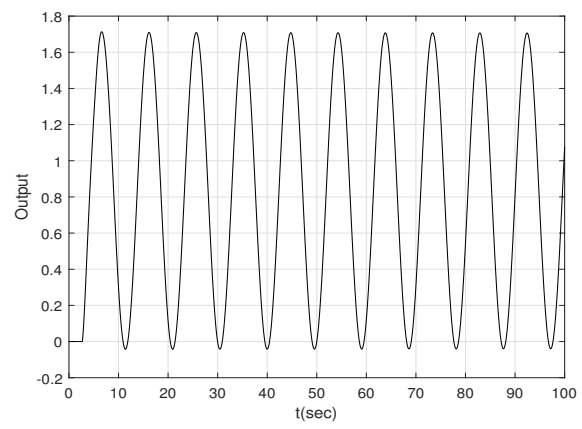

Figure 11. Unit step response of Example 3 for the second order approximation and the critical point $h=2.6962$.

that are already complicated for FOS have become easier. The examples show that the proposed method gives very reasonable results. For this purpose, integer-order approximations have been used. Thus, a fractional order equation has been turned into an integer-order one, and then the stability test has been applied to the system. When using integer-order approximations, there can be a difference depending on the degree of approximation. Studies have shown that good approximation results for FOS are obtained when using third or fourth-order approximations. Therefore, when determining the stability range of $h$, the order of approximation can cause some differences in the calculations. However, it can be said that the first order approximation is sufficient for determining whether a system is stable or unstable because higher order approximations make mathematical operations quite complicated. Besides, too large values of the time delay can produce unwanted results in system performance. Thus, it is necessary to investigate of stability range of $h$ to obtain reasonable results. For future works, stability analysis can be investigated for FOS having parameter uncertainty or different time delays. In addition, stability for different types of controllers can also be investigated. As there are no analytical methods in this area, the studies on this subject will contribute significantly to the field.

\section{References}

[1] Petráš, I. (2011). Stability test procedure for a certain class of the fractional-order systems.Proceedings of the 2011 12th International Carpathian Control Conference, ICCC'2011, (5), 303-307.

[2] Podlubny, I. (1999). Fractional-order systems and $P I^{\lambda} D^{\mu}$-controllers. IEEE Transactions on Automatic Control, 44(1), 208-214.
[3] Liu, M., Dassios I., Milano F. (2019). On the Stability Analysis of Systems of Neutral Delay Differential Equations. Circuits, Systems, and Signal Processing, 38(4), 1639-1653.

[4] Milano, F., Dassios, I. (2016). Small-Signal Stability Analysis for Non-Index 1 Hessenberg Form Systems of Delay Differential-Algebraic Equations. IEEE Transactions on Circuits and Systems I: Regular Papers, 63(9), 15211530.

[5] Liu, M., Dassios I., Tzounas, G., Milano F. (2019). Stability Analysis of Power Systems with Inclusion of Realistic-Modeling of WAMS Delays. IEEE Transactions on Power Systems, 34(1), 627-636.

[6] Petras, I. (2010). Fractional-Order Memristor-Based Chua's Circuit.IEEE Transactions on Circuits and Systems, 57(12), 975-979.

[7] Dorčák, L., Valsa, J., Gonzalez, E., Terpák, J., Petráš, I., \& Pivka, L. (2013). Analogue realization of fractional-order dynamical systems. Entropy, 15(10), 4199-4214.

[8] Shah, P., \& Agashe, S. (2016). Review of fractional PID controller. Mechatronics, 38, 2941.

[9] Podlubny, I., Petráš, I., O'Leary, P., Dorčák, L., \& Vinagre, B. M. (2002). Analogue realizations of fractional order controllers. Nonlinear dynamics, 29, 281-296.

[10] Luo, Y., \& Chen, Y. (2012). Stabilizing and robust fractional order PI controller synthesis for first order plus time delay systems. Automatica, 48(9), 2159-2167.

[11] Tang, X., Shi, Y., \& Wang, L. L. (2017). A new framework for solving fractional optimal control problems using fractional pseudospectral methods. Automatica, 78, 333-340.

[12] Razminia, A., \& Baleanu, D. (2013). Complete synchronization of commensurate fractional order chaotic systems using sliding mode control. Mechatronics, 23(7), 873-879. 
[13] Ozturk, N. (1995). Stability Independent of distributed Lag for A Special Class of Distributed Parameter Systems. In Proceedings of the 34th IEEE Conference on Decision and Control, 13-15 December, New Orleans, LA, USA, 3245-3246).

[14] Tan, N.,Özgüven, Ö. F., \& Özyetkin,M. M. (2009). Robust stability analysis of fractional order interval polynomials. ISA Transactions, 48(2), 166-172.

[15] Ozturk, N. and Uraz, A. (1985). An Analysis Stability Test for a Certain Class of Distributed Parameter Systems with Delays.IEEE Transactions on Circuits and Systems, CAS-32, 393-396.

[16] Petras, I. (2008). Stability of FractionalOrder Systems with Rational Orders.Fractional calculus and Applied Aalysis, $12(3), 25$.

[17] Sabatier, J., Moze, M., \& Farges, C. (2010). LMI stability conditions for fractional order systems. Computers and Mathematics with Applications, 59(5), 1594-1609.

[18] Matignon, D. (1996). Stability results for fractional differential equations with applications to control processing. Computational engineering in systems applications, 963-968.

[19] Matignon, D. (1998). Stability properties for generalized fractional differential systems. ESAIM: Proceedings Fractional Differential Systems: Models, Methods and Aplicaitons, (5), 145-158.

[20] Lazarević, M. P., \& Spasić, A. M. (2009). Finite-time stability analysis of fractional order time-delay systems: Gronwall's approach. Mathematical and Computer Modelling, 49(34), 475-481.

[21] Lazarević, M. P., \& Debeljković, D. L. (2008). Finite time stability analysis of linear autonomous fractional order systems with delayed state. Asian Journal of Control, 7(4), 440-447.

[22] Hwang, C., \& Cheng, Y. C. (2006). A numerical algorithm for stability testing of fractional delay systems. Automatica, 42(5), 825-831.

[23] Oustaloup, A., Melchior, P., Lanusse, P., Dancla, F., \& Crone, E. (2000). The CRONE toolbox for Matlab.In Proceedings of the 2000 IEEE International Symposium on Computer-Aided Control System Design. Anchorage, Alaska, USA September 25-27, 2000,190-195.

[24] Melchior, P., Orsoni, B., Oustaloup, A., Cnrs, U. M. R., \& Bordeaux, U. (2001). The CRONE toolbox for Matlab: Fractional Path Planning Design in Robotics.In Proceedings 10th IEEE International Workshop on Robot and Human Interactive Communication, ROMAN 2001 (Cat. No.01TH8591), 534-540.

[25] Costa, D. V. and J. S. da. (2004). NINTEGER: A Non-Integer Control Toolbox for MATLAB. In Proceedings of 1st IFAC Workshop on Fractional Differentation and Its Applications, Bordeaux, France, 1-6.

[26] Tepljakov, A., Petlenkov, E., \& Belikov, J. (2011). FOMCON: Fractional-order modeling and control toolbox for MATLAB.Proceedings of the 18th International Conference Mixed Design of Integrated Circuits and SystemsMIXDES 2011, (4), 684-689.

[27] Ozyetkin, M. M. (2018). A simple tuning method of fractional order $P I^{\lambda_{-}}$ $P D^{\mu}$ controllers for time delay systems. ISA Transactions, 74, 77-87.

[28] Özyetkin, M. M., Yeroğlu, C., Tan, N., \& Tağluk, M. E. (2010). Design of PI and PID controllers for fractional order time delay systems. IFAC Proceedings Volumes (IFACPapersOnline), 43, 355-360.

[29] Krishna, B. T. (2011). Studies on fractional order differentiators and integrators: A survey. Signal Processing, 91(3), 386-426.

[30] Chen, Y. Q., Petráš, I., \& Xue, D. (2009). Fractional order control - A tutorial. Proceedings of the American Control Conference, 1397-1411.

[31] Silva, G. J., Datta, A., \& Bhattacharyya, S. P. (2005). PID Controllers for Time-Delay Systems. Birkhauser Boston.

[32] Walton, K., \& Marshall, J. E. (1987). Direct method for TDS stability analysis.IEE Proceedings D Control Theory and Applications, 134(2), 101-107.

[33] Ozturk, N. (1988). Stability Intervals for Delay Systems.In Proceedings of the 27th Conference on Decision and Control, Austin, Texas, 2215-2216.

Münevver Mine Özyetkin received the Ph.D. degree in Electrical and Electronics Engineering Department from Inonu University. She was awarded a grant by TUBITAK (The Scientific and Technological Research Council of Turkey) to study insulin control for diabetic patients (artificial pancreas) between 2010 and 2011, at Clemson University, USA. She is interested in fractional order control systems, design of fractional order controllers, robust control, and stability analysis. She is currently an Assistant Prof. at Aydin Adnan Menderes University.

(10) http://orcid.org/0000-0002-3819-5240 
Dumitru Baleanu's research interests include fractional dynamics and its applications, fractional differential equations, discrete mathematics, dynamic systems on time scales, the wavelet method and its applications, quantization of the systems with constraints, Hamilton-Jacobi formalism, geometries admitting generic and non-generic symmetries. He has published more than 600 papers indexed in SCI. He is one of the editors of 5 books published by Springer. Dumitru is an editorial board member of the following journals indexed in SCI: Abstract and Applied Analysis, Central European Journal of Physics, Advances in
Difference Equations, Scientific Research and Essays (SRE) and Fractional Calculus and Applied Analysis. He is also an editorial board member of 12 different journals which are not indexed in SCI. He is currently a faculty member of Department of Mathematics and Computer Sciences at Cankaya University, Ankara, Turkey. Besides, he is a Professor at Institute of Space Sciences, Magurele-Bucharest, Romania

(iD http://orcid.org/0000-0002-0286-7244

An International Journal of Optimization and Control: Theories \& Applications (http://ijocta.balikesir.edu.tr)

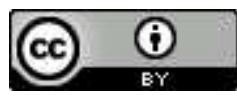

This work is licensed under a Creative Commons Attribution 4.0 International License. The authors retain ownership of the copyright for their article, but they allow anyone to download, reuse, reprint, modify, distribute, and/or copy articles in IJOCTA, so long as the original authors and source are credited. To see the complete license contents, please visit http://creativecommons.org/licenses/by/4.0/. 